\title{
A Comparison of Hierarchical and Non-Hierarchical Bayesian Approaches for Fitting Allometric Larch (Larix.spp.) Biomass Equations
}

\author{
Dongsheng Chen ${ }^{1,+}$, Xingzhao Huang ${ }^{2,+}$, Xiaomei Sun ${ }^{1}$, Wu Ma ${ }^{3}$ and Shougong Zhang ${ }^{1, *}$ \\ Received: 9 September 2015 ; Accepted: 18 December 2015 ; Published: date \\ Academic Editor: Eric J. Jokela \\ 1 Key Laboratory of Tree Breeding and Cultivation, State Forestry Administration, Research Institute of \\ Forestry, Chinese Academy of Forestry,Beijing 100091, China;nefucds@126.com (D.C.); \\ xmsun@caf.ac.cn (X.S.) \\ 2 School of Forestry \& Landscape of Architecture, Anhui Agricultural University, Hefei 230036, China; \\ xingzhaoh@163.com \\ 3 School of Natural Resources, West Virginia University, Morgantown, WV 26506, USA; \\ wuma@mix.wvu.edu \\ * Correspondence: shougong.zhang@caf.ac.cn; Tel./Fax: +86-10-6288-9008 \\ + These authors contributed equally to this work.
}

\begin{abstract}
Accurate biomass estimations are important for assessing and monitoring forest carbon storage. Bayesian theory has been widely applied to tree biomass models. Recently, a hierarchical Bayesian approach has received increasing attention for improving biomass models. In this study, tree biomass data were obtained by sampling 310 trees from 209 permanent sample plots from larch plantations in six regions across China. Non-hierarchical and hierarchical Bayesian approaches were used to model allometric biomass equations. We found that the total, root, stem wood, stem bark, branch and foliage biomass model relationships were statistically significant ( $p$-values $<0.001)$ for both the non-hierarchical and hierarchical Bayesian approaches, but the hierarchical Bayesian approach increased the goodness-of-fit statistics over the non-hierarchical Bayesian approach. The $R^{2}$ values of the hierarchical approach were higher than those of the non-hierarchical approach by $0.008,0.018,0.020,0.003,0.088$ and 0.116 for the total tree, root, stem wood, stem bark, branch and foliage models, respectively. The hierarchical Bayesian approach significantly improved the accuracy of the biomass model (except for the stem bark) and can reflect regional differences by using random parameters to improve the regional scale model accuracy.
\end{abstract}

Keywords:larch; non-hierarchical Bayesian approach; hierarchical Bayesian approach; biomass model

\section{Introduction}

Larch (Larixspp.) is a commercially valuable timber that is widely planted in the mountains of North, Northeast and Southwest China because of its straight shape and high resistance to bending and cracking. Chinese larch plantations comprise approximately 3.14 million ha, accounting for $6.66 \%$ of all timber plantations, with a volume of approximately 18.4 million $\mathrm{m}^{3}$, accounting for $7.42 \%$ of the total plantation volume. China contains the largest area of larch plantations in the world [1].

The plantation biomass and carbon sequestration calculations have been studied by numerous researchers [2-4]. The calculations are a prerequisite for understanding carbon pool dynamics in plantations. Allometric equations are commonly used to quantify plant biomass based on the relationship between tree biomass and diameter [5-7]. The biomass and diameter data sets are typically collected from sample plots in the field. This technique is generally destructive, 
labour-intensive and time-consuming [8]. Established allometric equations can be applied to quantify and monitor tree biomass, as tree diameter can be directly measured in the field.

Selecting the appropriate estimation technique is critical for accurate biomass estimations. Most studies estimate allometric equation parameters using ordinary least-squares or maximum-likelihood methods, which represent a classic statistical approach. Mauricio et al. [9] applied Bayesian methods to estimate aboveground tree biomass using data from six trees, producing similar fitting results as the classic statistical method that used data from 40 to 60 trees. Zhang et al. [10] confirmed that the Bayesian method with informative priors outperformed non-informative priors and the classic statistical approach.Bayesian estimates of allometric equations may be effectively applied in one location, but produce significantly different results when applied elsewhere [11,12]. In recent years, the random variations between geographical locations [13] or among individual samples [14] have gained increasing attention. However, allometric equations based on traditional statistical methods ignore regional variations $[15,16]$.

A hierarchical Bayesian approach can incorporate regional variations during the model fitting process $[17,18]$. When data are obtained from multiple regions, the hierarchical Bayesian approach assumes that subjects (e.g., trees) in the same spatial region share common attributes [19]. This approach allows for the estimation of a very broad range of equations and can yield more realistic assessments of parameter estimate uncertainties[18,20-22]. The hierarchical Bayesian approach has been applied to forestry [23-25], but has rarely been used to establish a regional scale biomass model. In this study, we applied a hierarchical Bayesian approach to fit allometric biomass equations and compared non-hierarchical and hierarchical Bayesian approaches for estimating the biomass in China's larch plantations.

\section{Materials and Methods}

\subsection{Study Sites}

The biomass data were collected from six different larch plantation regions in China (Figure 1, Table1). The experimental sites in this study encompassed the main timber production larch plantation regions in China. L1 is the Changlinggang Forest Farm $\left(30^{\circ} 48^{\prime} \mathrm{N}, 110^{\circ} 02^{\prime} \mathrm{E}\right)$ in Jianshi County, Hubei Province, in the northern subtropical region, which is dominated by Japanese larch(Larixkaempferi Carr). L2 is the Xiaolongshan Research Institute of Forestry ( $34^{\circ} 09^{\prime} \mathrm{N}, 105^{\circ} 52^{\prime} \mathrm{E}$ ) in Tianshui City, Gansu Province, in the warm-temperate region, which is also dominated by Japanese larch. L3 is the MulanWeichang National Forestry Administration Bureau (41 $\left.{ }^{\circ} 43^{\prime} \mathrm{N}, 118^{\circ} 7^{\prime} \mathrm{E}\right)$ in Weichang County, Hebei Province, which is dominated by North Chineselarch(Larixprincipisrupprechtii Mayr). L4 is the Dagujia Forest Farm ( $\left.42^{\circ} 21^{\prime} \mathrm{N}, 124^{\circ} 52^{\prime} \mathrm{E}\right)$ in Qingyuan County, Liaoning Province, which is dominated by Japanese larch. Both L3 and L4 are located in a temperate region. L5 is the Mengjiagang Forest Farm $\left(46^{\circ} 32^{\prime} \mathrm{N}, 129^{\circ} 10^{\prime}\right.$ E) in Jiamusi City, Heilongjiang Province, which is dominated by Korean larch (Larixolgensis Henry). L6 is the Wuerqihan Forestry Bureau $\left(49^{\circ} 34^{\prime} \mathrm{N}, 121^{\circ} 25^{\prime}\right.$ E) in Yakeshi City, Inner Mongolia, which is dominated by Chinese larch (Larixgmelini Kuzen). Both L5 and L6 are located in a cold-temperate region. These locations span the majority of the larch plantation areas in China.

Table 1. Six larch plantation study regions.

\begin{tabular}{ccccccc}
\hline \multirow{2}{*}{ Regions } & \multirow{2}{*}{ Species } & \multirow{2}{*}{ Plots } & \multicolumn{2}{c}{ Location } & Altitude & Sample \\
\cline { 4 - 5 } & & & Longitude (E) & Latitude (N) & (m) & Trees \\
\hline L1 & L. kaempferi & 34 & $109^{\circ} 21^{\prime} \sim 111^{\circ} 07^{\prime}$ & $29^{\circ} 05^{\prime} \sim 31^{\circ} 20^{\prime}$ & $1800 \sim 2500$ & 40 \\
L2 & L. kaempferi & 33 & $105^{\circ} 48^{\prime} \sim 106^{\circ} 05^{\prime}$ & $34^{\circ} 09^{\prime} \sim 34^{\circ} 16^{\prime}$ & $800 \sim 1600$ & 60 \\
L3 & L. principis-rupprechtii & 36 & $116^{\circ} 32^{\prime} \sim 117^{\circ} 14^{\prime}$ & $41^{\circ} 35^{\prime} \sim 42^{\circ} 40^{\prime}$ & $1200 \sim 1800$ & 62 \\
L4 & L. kaempferi & 36 & $124^{\circ} 50^{\prime} \sim 125^{\circ} 10^{\prime}$ & $40^{\circ} 50^{\prime} \sim 42^{\circ} 22^{\prime}$ & $300 \sim 700$ & 60 \\
L5 & L. olgensis & 34 & $128^{\circ} 55^{\prime} \sim 129^{\circ} 15^{\prime}$ & $45^{\circ} 31^{\prime} \sim 46^{\circ} 49^{\prime}$ & $200 \sim 800$ & 44 \\
L6 & L. gmelini & 36 & $123^{\circ} 36^{\prime} \sim 125^{\circ} 19^{\prime}$ & $51^{\circ} 32^{\prime} \sim 52^{\circ} 20^{\prime}$ & $400 \sim 900$ & 44 \\
\hline
\end{tabular}




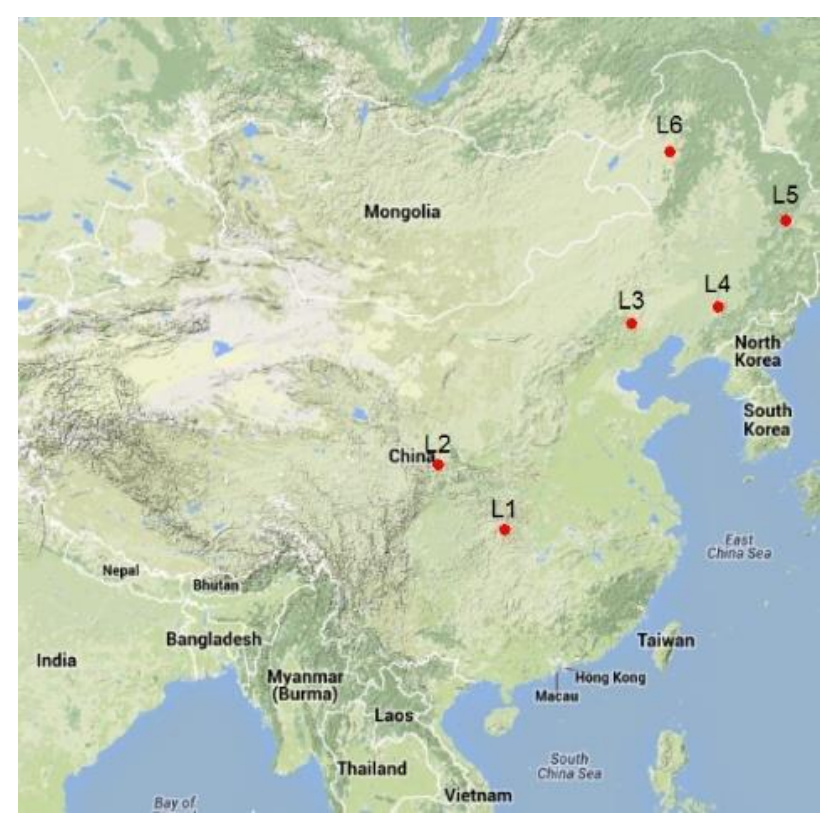

Figure 1. Six larch plantation regions in China.

We randomly selected 310 trees based on the diameter classes in each plot. Selected trees were felled. Tree height, diameter at breast height (DBH), crown length and crown width were measured and recorded. Each crown was classified into three classes (top, middle and bottom), and all live and dead branches from each canopy class were removed and weighed. Three branches of each canopy class were selected, and their foliage and small branches were removed. The stem was cut into 1-m-long sections and weighed. Then, we took discs from the stem at each cut and separated the stem wood from the bark. Roots were manually excavated from the soil surface to their ends along the direction of root growth to measure the belowground biomass. All excavated roots were washed and sorted into three diameter classes: large $(>5.0 \mathrm{~cm})$, medium $(2.0-5.0 \mathrm{~cm})$ and small $(<2.0 \mathrm{~cm})$. We also measured the fresh biomass of each part of the tree, including branches, foliage, stems, bark and roots. These subsamples were transported to the laboratory for analysis.

All subsamples were dried at $80^{\circ} \mathrm{C}$ and weighed to determine the dry biomass percentage for each part of the tree. The dry weight was calculated as the fresh weight of each part multiplied by the corresponding dry biomass percentage, while the total dry biomass for the tree was determined by summing the dry weights of different parts of the sampled tree (Table 2).

Table 2. Descriptive statistics of trees sampled for fitting the biomass equations (Std, standard deviation).

\begin{tabular}{|c|c|c|c|c|c|c|c|c|c|c|c|c|c|c|}
\hline \multirow{2}{*}{ Regions } & \multicolumn{2}{|c|}{$\mathrm{DBH}(\mathrm{cm})$} & \multicolumn{2}{|c|}{$\begin{array}{c}\text { Stem Wood } \\
(\mathrm{Kg})\end{array}$} & \multicolumn{2}{|c|}{$\begin{array}{l}\text { Stem Bark } \\
(\mathrm{Kg})\end{array}$} & \multicolumn{2}{|c|}{ Branch $(\mathrm{Kg})$} & \multicolumn{2}{|c|}{ Foliage (Kg) } & \multicolumn{2}{|c|}{$\operatorname{Root}(\mathrm{Kg})$} & \multicolumn{2}{|c|}{ Total(Kg) } \\
\hline & Mean & Std & Mean & Std & Mean & Std & Mean & Std & Mean & Std & Mean & Std & Mean & Std \\
\hline L1 & 16.5 & 5.9 & 68.9 & 53.6 & 9.9 & 7.7 & 10.9 & 6.0 & 3.8 & 2.9 & 23.6 & 12.3 & 117.2 & 80.9 \\
\hline L2 & 13.0 & 6.6 & 38.7 & 45.9 & 5.7 & 5.8 & 7.0 & 6.7 & 2.3 & 2.2 & 13.2 & 16.3 & 77.0 & 80.2 \\
\hline L3 & 11.5 & 4.6 & 35.1 & 35.0 & 5.2 & 5.1 & 13.2 & 14.1 & 3.4 & 3.5 & 11.2 & 12.7 & 99.5 & 68.3 \\
\hline L4 & 14.5 & 5.5 & 78.4 & 81.9 & 8.5 & 7.2 & 8.4 & 6.8 & 3.5 & 2.7 & 18.4 & 19.6 & 155.1 & 115.8 \\
\hline L5 & 17.8 & 4.6 & 87.1 & 54.6 & 9.5 & 4.9 & 10.7 & 5.0 & 3.3 & 1.2 & 20.5 & 13.9 & 146.4 & 69.3 \\
\hline L6 & 12.4 & 6.0 & 55.6 & 60.9 & 6.5 & 6.3 & 6.3 & 6.7 & 1.6 & 1.6 & 20.3 & 21.0 & 73.0 & 74.5 \\
\hline
\end{tabular}

\subsection{Bayesian Approach}

By modelling the observed data and unobserved variables, regions can be regarded as random variables. The Bayesian approach provides a cohesive framework for combining hierarchical data models and external knowledge [22,26]. The Bayesian method is a statistical framework based on combining data with prior information about parameter values to derive probabilities of the various parameter values $[27,28]$. In our analysis, the distributional model $f(y \mid \theta)$ represents the biomass data 
$y=\left(y_{1}, \ldots, y_{j}\right)$ given a parameter vector $\theta=\left(\theta_{1}, \ldots, \theta_{j}\right)$. Then, $\pi(\theta \mid \lambda)$ is determined, where $\lambda$ is a hyperparameter vector[29]. The inference parameter $\theta$ is based on its posterior distribution:

$$
p(\theta \mid y, \lambda)=\frac{p(y, \theta \mid \lambda)}{\int p(y, \theta \mid \lambda) d \theta}=\frac{f(y \mid \theta) \pi(\theta \mid \lambda)}{\int f(y \mid \theta) \pi(\theta \mid \lambda) d \theta}
$$

This posterior distribution is used for a Bayesian statistical inference, in contrast to the Frequentist method, which uses $f(y \mid \theta)$ for inference. The $f(y \mid \theta)$ provides the distribution of $y$ assuming $\theta$ is known, which is considered a likelihood function when viewed as a function of the parameters. The prior distributions of $\pi(\theta \mid \lambda)$ can be obtained from parameters reported in the literatureor using vague priors.

\subsection{AllometricModels}

Numerous models have been developed for estimating tree biomass, especially based on the allometric equations: $W=a D B H^{b}$ and $W=a\left(D B H^{2} H\right)^{b}$ (where $W$ is the tree biomass, $D B H$ is the diameter at breast height, and $H$ is the tree height). $D B H$ is often used in biomass equations $[11,30,31]$ and is more easily obtained than $H$. Therefore, $W=a D B H^{b}$ was applied as the biomass model in this study. However, a heteroscedasticity exists when directly fitting tree biomass. Typically, logarithms $(\ln (W)=\ln (a+b) \ln (D B H))$ can counteract heteroscedasticity [32]. Thus, the total tree, root, stem wood, stem bark, branch and foliage biomasses were modelled using the following log-transformed allometric equation:

$$
y_{i}=a+b x_{i}+e_{i}
$$

Where $y_{i}$ is the log-transformed biomass of each part of theith sampled tree, $x_{i}$ is the log-transformed $\mathrm{DBH}$ of the $i$ th sampled tree, and $a$ and $b$ are the intercept and slope, respectively. The error term $e_{i}$ assumes a normal distribution with a mean of zero and constant variance ${ }^{2}$.

\subsection{Modelling Approaches}

\subsubsection{Non-Hierarchical Bayesian Approach}

The non-hierarchical Bayesian structure is shown in Figure 2.The observed values $x_{i}$ are shown at the bottom. $\theta$ represents the unknown parameters of $a$ and $b$ associated with probability distribution $f(y \mid \theta)$. In the non-hierarchical Bayesian approach, the parameters in Equation (2) are treated as random variables. This approach was used to fit Equation (2), as given by:

$$
\begin{aligned}
& p(a, b \mid d a t a)=\prod_{i} \frac{1}{\sqrt{2 \pi} \sigma^{2}} \exp \left(\frac{-\left(y_{i}-g\left(x_{i}: a, b\right)\right)^{2}}{2 \sigma^{2}}\right) \\
& \begin{array}{llllll}
\theta_{1} & \theta_{2} & \ldots & \ldots & \ldots & \theta_{j}
\end{array} \\
& \begin{array}{llllll}
x_{1} & x_{2} & \ldots & \ldots & x_{i}
\end{array}
\end{aligned}
$$
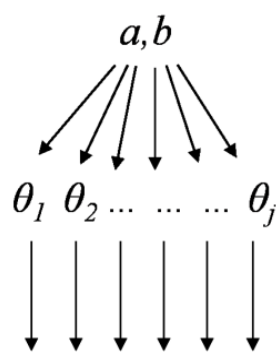

Figure 2. Bayesian non-hierarchical structure. 


\subsubsection{Hierarchical Bayesian Approach}

Our data were collected fromsix different spatial regions and four species, which exhibit typical hierarchical data characteristics (Table 1). Figure 3 summarizes the hierarchical Bayesian approach. The biomass data can be used to estimate parameter $\theta_{i}$ for each region. Thus, the allometric equation for the hierarchical Bayesian approach can be written as follows:

$$
y_{j(i)}=a_{j}+b_{j} x_{j(i)}+e_{i}
$$

Where $y_{j(i)}$ and $x_{j(i)}$ are the log-transformed biomass $y$ and $D B H$ of the $i$ th tree in the $j$ th region, respectively, and $a_{j}$ and $b_{j}$ are the intercept and slope of the $j$ th region.The error term $e_{i}$ assumes a normal distribution with a mean of zero and constant variance.

Our analysis hierarchically interprets the parameter estimation problem using cross-regional biomass data (Figure 3). Parameters $a_{j}$ and $b_{j}$ have specific values for each region, allowing for polymorphic lines and multiple asymptotes. For the $j$ th region of theith tree, the parameter $\theta_{j}$ in Equation (4) is defined as:

$$
\theta_{j}=\left(a_{j}, b_{j}\right)=\left[\begin{array}{l}
a+a_{i} \\
b+b_{i}
\end{array}\right]
$$

The hierarchical Bayesian approach is used to fit Equation (4), as given by:

$$
p(a, b \mid \text { data })=p(a, b) \prod_{j=1}^{J} \frac{\Gamma(a+b)}{\Gamma(a) \Gamma(b)} \frac{\Gamma\left(a+y_{j}\right) \Gamma\left(b+n_{j}-y_{j}\right)}{\Gamma\left(a+b+n_{j}\right)}
$$

Where $n_{j}$ is the number of regions.

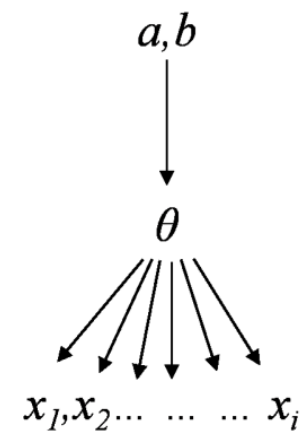

Figure 3.Bayesian hierarchical structure.

\subsection{PriorParameter Distributions}

The choice of prior distributions for each parameter is critical in the Bayesian method [33]. Zhang et al.[10] found that Bayesian analyses with non-informative priors and a classic statistical approach yielded results that were similar to using parameters and statistics to fit allometric biomass equations. However, the Bayesian method with informative priors performed better than the non-informative priors and classic statistical approach. Thus, the appropriate prior distribution selections for all parameters are critical for improving the model precision. The prior distribution information can be obtained from parameters reported in the literature. In this study, the prior distributions of $a$ and $b$ (total tree, root, stem wood, stem bark, branch and foliage) were obtained for 36 biomass equations from 6 Chinese larch publications (Table S1). We assumed that $a$ and $b$ follow a bivariate normal distribution $N(\mu, \Sigma)$, where $\mu\left(\mu_{a}, \mu_{b}\right)$ is a vector of means and $\Sigma$ is the covariance matrix (Table 3). 
Table 3. Prior parameter distributions from the published literature for each equation.

\begin{tabular}{cccc}
\hline Component & $\mu_{a}$ & $\mu_{b}$ & \multicolumn{2}{c}{$\Sigma$} \\
\hline Total tree & -1.834 & 0.843 & $\left(\begin{array}{ll}0.248 & -0.032 \\
-0.032 & 0.006\end{array}\right)$ \\
Root & -3.769 & 0.856 & $\left(\begin{array}{ll}0.232 & -0.302 \\
-0.302 & 0.060\end{array}\right)$ \\
Stem wood & -2.649 & 0.888 & $\left(\begin{array}{ll}0.214 & -0.022 \\
-0.022 & 0.004\end{array}\right)$ \\
Stem bark & -3.539 & 0.694 & $\left(\begin{array}{ll}1.056 & -0.149 \\
-0.149 & 0.031\end{array}\right)$ \\
Branch & -3.113 & 0.641 & $\left(\begin{array}{ll}2.370 & -0.375 \\
-0.375 & 0.089\end{array}\right)$ \\
Foliage & -3.719 & 0.597 & $\left(\begin{array}{ll}2.608 & -0.303 \\
-0.303 & 0.056\end{array}\right)$ \\
\hline
\end{tabular}

\subsection{Model Fitting}

Using the non-hierarchical Bayesian approach as a base method, we used the nonlinear extra sum of squares method and the Lakkis-Jones test to assess whether the hierarchical Bayesian approach significantly improved the accuracy of the biomass equation [34,35]. The statistics are given by thenonlinear extra sum of squares:

$$
F=\left(\frac{S S E_{N}-S S E_{B}}{d f_{N}-d f_{B}}\right) \frac{d f_{B}}{S S E_{B}}
$$

And Lakkis-Jones test:

$$
L=2 \operatorname{Ln}\left(\left(\frac{S S E_{N}}{S S E_{B}}\right)^{\frac{n}{2}}\right)
$$

where $S S E_{N}$ is the sum of squares of residuals in the non-hierarchical Bayesian approach, $S S E_{B}$ is the sum of squares of residuals in the hierarchical Bayesian approach, $d f_{N}$ and $d f_{B}$ are the degrees of freedom of the non-hierarchical and hierarchical Bayesian approaches, respectively, and $n$ is the number of observations used in the model fitting. The F-statistic follows an F-distribution, and the $L$-statistic follows a $\chi^{2}$-distribution with $v=d f_{N}-d f_{B}$ degrees of freedom.

The Markov Chain Monte Carlo (MCMC) algorithm was used to estimate model parameters in both non-hierarchical and hierarchical Bayesian approaches. All models were fitted using the MCMC method in the MCMCglmm package and R2WinBUGS package in R version 3.1.1 [36,37].

\section{Results}

\subsection{Fitted Biomass Models}

This study compiled 36 logarithmic biomass equations for larch biomass in China. The prior parameter distributions were obtained from the published literature. Parameters $a$ and $b$ followed bivariate normal distributions in each component biomass model (Table 3). Based on the Bayesian theory with informative priors, we obtained the posterior probability distributions of the two parameters for each component biomass model. The values of $a$ and $b$ for the total tree and 
component biomass models were estimated using non-hierarchical and hierarchical Bayesian approaches.

The posterior total tree biomassprobability distributions are shown in Figure 4, which are similar to the posterior probability distributions of the component biomass model. According to the fitted results, the total tree, root, stem wood, stem bark, branch and foliage ( $p$-values $<0.001$ ) biomass model relationships were significant for both the non-hierarchical and hierarchical Bayesian approaches. 10,000 iterations were performed for each model fitting to ensure convergence and obtain posterior distributions of the estimated parameters. Of these, the first 500 were discarded as burn-in iterations. The thinning parameter between the non-hierarchical and hierarchical approaches was set at three chains to reduce the impact of the correlation between neighbouring iterations. The standard deviation (S.D.) and $P_{2.5 \%}-P_{97.5 \%}$ were then calculated based on the samples. The parameter estimates using the non-hierarchical and hierarchical Bayesian approaches are presented in Tables 4 and 5.

Table 4. Tree biomass model parameters using the Bayesian non-hierarchical approach.

\begin{tabular}{ccccc}
\hline Component & Parameters & Mean & S.D. & $\boldsymbol{P}_{2.5 \%}-\boldsymbol{P}_{\mathbf{9 7 . 5} \%}$ \\
\hline \multirow{2}{*}{ Total tree } & $a$ & -2.117 & 0.054 & $(-2.225-2.012)$ \\
& $b$ & 2.42 & 0.021 & $(2.3792 .462)$ \\
\hline \multirow{2}{*}{ Root } & $a$ & -3.963 & 0.092 & $(-4.146-3.784)$ \\
& $b$ & 2.459 & 0.036 & $(2.3892 .530)$ \\
\hline \multirow{2}{*}{ Stem wood } & $a$ & -3.521 & 0.081 & $(-3.684-3.362)$ \\
& $b$ & 2.728 & 0.032 & $(2.6662 .791)$ \\
\hline \multirow{2}{*}{ Stem bark } & $a$ & -3.927 & 0.093 & $(-4.113-3.746)$ \\
& $b$ & 2.152 & 0.036 & $(2.0182 .224)$ \\
\hline \multirow{2}{*}{ Branch } & $a$ & -2.682 & 0.15 & $(-2.225-2.012)$ \\
& $b$ & 1.783 & 0.059 & $(-2.225-2.012)$ \\
\hline \multirow{2}{*}{ Foliage } & $a$ & -3.28 & 0.176 & $(-3.631-2.937)$ \\
& $b$ & 1.578 & 0.069 & $(1.4441 .715)$ \\
\hline
\end{tabular}

Table 5. Tree biomass model parameters using the Bayesian hierarchical approach $\left(a_{1}-a_{6}\right.$ and $b_{1}-b_{6}$ represent the six regions from L1-L6).

\begin{tabular}{ccccccc}
\hline \multirow{2}{*}{ Parameters } & \multicolumn{3}{c}{ Total Tree } & \multicolumn{3}{c}{ Root } \\
\cline { 2 - 7 } & Mean & S.D & $\boldsymbol{P}_{\mathbf{2 . 5} \%}-\boldsymbol{P}_{\mathbf{9 7 . 5} \%}$ & Mean & S.D & $\boldsymbol{P}_{2.5 \%}-\boldsymbol{P}_{97.5 \%}$ \\
\hline$a_{1}$ & -1.878 & 0.159 & $(-2.173-1.554)$ & -2.041 & 0.314 & $(-2.660-1.417)$ \\
$a_{2}$ & -1.849 & 0.076 & $(-1.994-1.700)$ & -3.929 & 0.133 & $(-4.191-3.671)$ \\
$a_{3}$ & -2.298 & 0.095 & $(-2.485-2.113)$ & -4.653 & 0.170 & $(-4.981-4.318)$ \\
$a_{4}$ & -2.650 & 0.137 & $(-2.919-2.381)$ & -4.412 & 0.222 & $(-4.842-3.991)$ \\
$a_{5}$ & -1.451 & 0.134 & $(-1.712-1.190)$ & -3.135 & 0.219 & $(-3.563-2.704)$ \\
$a_{6}$ & -2.540 & 0.104 & $(-2.739-2.330)$ & -4.030 & 0.186 & $(-4.399-3.670)$ \\
$b_{1}$ & 2.307 & 0.058 & $(2.1882 .413)$ & 1.829 & 0.114 & $(1.6042 .053)$ \\
$b_{2}$ & 2.277 & 0.032 & $(2.2142 .338)$ & 2.434 & 0.056 & $(2.3252 .543)$ \\
$b_{3}$ & 2.519 & 0.040 & $(2.4422 .598)$ & 2.705 & 0.071 & $(2.5632 .843)$ \\
$b_{4}$ & 2.649 & 0.052 & $(2.5462 .752)$ & 2.600 & 0.084 & $(2.4392 .763)$ \\
$b_{5}$ & 2.208 & 0.049 & $(2.1132 .304)$ & 2.138 & 0.080 & $(1.9802 .295)$ \\
$b_{6}$ & 2.554 & 0.041 & $(2.4712 .635)$ & 2.551 & 0.074 & $(2.4062 .698)$ \\
\hline
\end{tabular}


Table 5.Cont

\begin{tabular}{ccccccc}
\hline \multirow{2}{*}{ Parameters } & \multicolumn{3}{c}{ Stem Wood } & \multicolumn{3}{c}{ Stem Bark } \\
\cline { 2 - 7 } & Mean & S.D & $\boldsymbol{P}_{2.5 \%}-\boldsymbol{P}_{97.5 \%}$ & Mean & S.D & $\boldsymbol{P}_{2.5 \%}-\boldsymbol{P}_{97.5 \%}$ \\
\hline$a_{1}$ & -4.394 & 0.250 & $(-4.894-3.915)$ & -3.971 & 0.134 & $(-4.251-3.741)$ \\
$a_{2}$ & -3.114 & 0.122 & $(-3.356-2.880)$ & -3.927 & 0.102 & $(-4.119-3.721)$ \\
$a_{3}$ & -3.356 & 0.152 & $(-3.649-3.057)$ & -3.904 & 0.106 & $(-4.105-3.684)$ \\
$a_{4}$ & -4.284 & 0.210 & $(-4.687-3.863)$ & -3.916 & 0.118 & $(-4.151-3.685)$ \\
$a_{5}$ & -3.007 & 0.195 & $(-3.402-2.634)$ & -3.897 & 0.115 & $(-4.110-3.657)$ \\
$a_{6}$ & -3.843 & 0.153 & $(-4.143-3.542)$ & -3.965 & 0.111 & $(-4.185-3.751)$ \\
$b_{1}$ & 2.971 & 0.091 & $(2.7963 .153)$ & 2.151 & 0.048 & $(2.0672 .247)$ \\
$b_{2}$ & 2.525 & 0.051 & $(2.4272 .628)$ & 2.146 & 0.042 & $(2.0622 .226)$ \\
$b_{3}$ & 2.671 & 0.063 & $(2.5462 .794)$ & 2.150 & 0.043 & $(2.0622 .231)$ \\
$b_{4}$ & 3.061 & 0.080 & $(2.9003 .214)$ & 2.166 & 0.046 & $(2.0782 .261)$ \\
$b_{5}$ & 2.597 & 0.071 & $(2.4622 .742)$ & 2.153 & 0.042 & $(2.0672 .232)$ \\
$b_{6}$ & 2.836 & 0.061 & $(2.7142 .955)$ & 2.142 & 0.043 & $(2.0572 .225)$ \\
\hline \multirow{2}{*}{ Parameters } & \multicolumn{9}{c}{ Branch } & & Foliage \\
\cline { 2 - 7 } & Mean & S.D & $\boldsymbol{P}_{2.5 \%}-\boldsymbol{P}_{97.5 \%}$ & Mean & S.D & $\boldsymbol{P}_{2.5 \%}-\boldsymbol{P}_{97.5 \%}$ \\
\hline$a_{1}$ & -2.374 & 0.381 & $(-3.080-1.599)$ & -2.867 & 0.477 & $(-3.796-1.928)$ \\
$a_{2}$ & -2.757 & 0.192 & $(-3.130-2.376)$ & -2.958 & 0.258 & $(-3.472-2.466)$ \\
$a_{3}$ & -3.387 & 0.282 & $(-3.931-2.818)$ & -4.702 & 0.337 & $(-5.358-4.049)$ \\
$a_{4}$ & -2.354 & 0.322 & $(-2.958-1.705)$ & -2.274 & 0.421 & $(-3.119-1.471)$ \\
$a_{5}$ & -1.989 & 0.343 & $(-2.666-1.331)$ & -1.659 & 0.422 & $(-2.473-0.843)$ \\
$a_{6}$ & -3.277 & 0.255 & $(-3.783-2.787)$ & -4.225 & 0.339 & $(-4.894-3.558)$ \\
$b_{1}$ & 1.673 & 0.138 & $(1.3921 .928)$ & 1.434 & 0.173 & $(1.0931 .771)$ \\
$b_{2}$ & 1.794 & 0.081 & $(1.6331 .948)$ & 1.429 & 0.108 & $(1.2211 .649)$ \\
$b_{3}$ & 2.287 & 0.118 & $(2.0452 .515)$ & 2.268 & 0.141 & $(1.9942 .539)$ \\
$b_{4}$ & 1.605 & 0.123 & $(1.3591 .835)$ & 1.263 & 0.161 & $(0.9521 .538)$ \\
$b_{5}$ & 1.543 & 0.125 & $(1.3031 .791)$ & 1.020 & 0.154 & $(0.7211 .326)$ \\
$b_{6}$ & 1.835 & 0.101 & $(1.6402 .036)$ & 1.717 & 0.135 & $(1.4541 .983)$ \\
\hline & \multicolumn{9}{c}{} & & &
\end{tabular}

Total
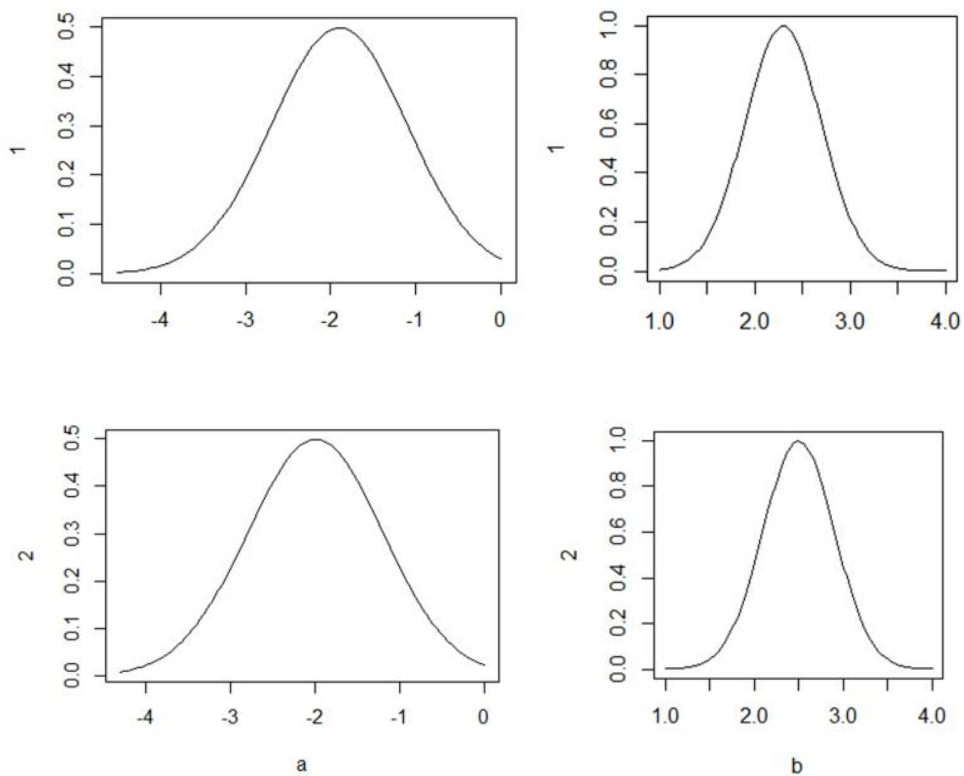

Figure 4. Posterior probability densities of two parameters for each total tree biomass model. 1 is the non-hierarchical approach, and 2 is the Bayesian hierarchical approach. 


\subsection{Comparison of Two BayesianApproaches}

The $p$-values, $R^{2}$, nonlinear extra sum of squares ( $F$-value) and the Lakkis-Jones ( $L$-value) tests of the biomass model estimated by the non-hierarchical and hierarchical Bayesian approaches are shown in Table 6. We detected significant differences between the two Bayesian approaches with respect to the stem wood, foliage, branch, root and total tree biomass models ( $p$-value $<0.001$ ). The hierarchical Bayesian approach increased the goodness-of-fit statistics. The $R^{2}$ values of the total tree, root, stem wood, stem bark, branch and foliage biomass models using the hierarchical Bayesian method were $0.008,0.018,0.020,0.003,0.088$ and 0.116 higher than non-hierarchical model, respectively.

Table 6.Evaluation of the non-hierarchical and hierarchical Bayesian approaches (1 and 2 represent the non-hierarchical and hierarchical Bayesian approaches, respectively).

\begin{tabular}{|c|c|c|c|c|c|c|c|}
\hline Component & Approach & $p$-Values & $R^{2}$ & F-Values & $\operatorname{Pr}>|F|$ & L-Values & $\operatorname{Pr}>|L|$ \\
\hline \multirow{2}{*}{ Total tree } & 1 & $<0.001$ & 0.981 & & & & \\
\hline & 2 & $<0.001$ & 0.989 & 7.071 & $<0.001$ & 154.386 & $<0.001$ \\
\hline \multirow{2}{*}{ Root } & 1 & $<0.001$ & 0.950 & & & & \\
\hline & 2 & $<0.001$ & 0.968 & 6.097 & $<0.001$ & 137.493 & $<0.001$ \\
\hline \multirow{2}{*}{ Stem wood } & 1 & $<0.001$ & 0.967 & & & & \\
\hline & 2 & $<0.001$ & 0.987 & 5.561 & $<0.001$ & 127.443 & $<0.001$ \\
\hline \multirow{2}{*}{ Stem bark } & 1 & $<0.001$ & 0.934 & & & & \\
\hline & 2 & $<0.001$ & 0.937 & 0.392 & 0.264 & 10.354 & 0.264 \\
\hline \multirow{2}{*}{ Branch } & 1 & $<0.001$ & 0.791 & & & & \\
\hline & 2 & $<0.001$ & 0.879 & 7.575 & $<0.001$ & 136.152 & $<0.001$ \\
\hline \multirow{2}{*}{ Foliage } & 1 & $<0.001$ & 0.682 & & & & \\
\hline & 2 & $<0.001$ & 0.798 & 6.039 & $<0.001$ & 165.496 & $<0.001$ \\
\hline
\end{tabular}

The performance of all fitted models is shown in Figure 5. Boxplots illustrate the residual tendency of each biomass model component for the two Bayesian approaches in each region. The hierarchical Bayesian approach residuals are closer to both the zero-line and the observed values compared to those of the non-hierarchical Bayesian approach. Thus, the hierarchical approach yielded more accurate parameter estimates. 

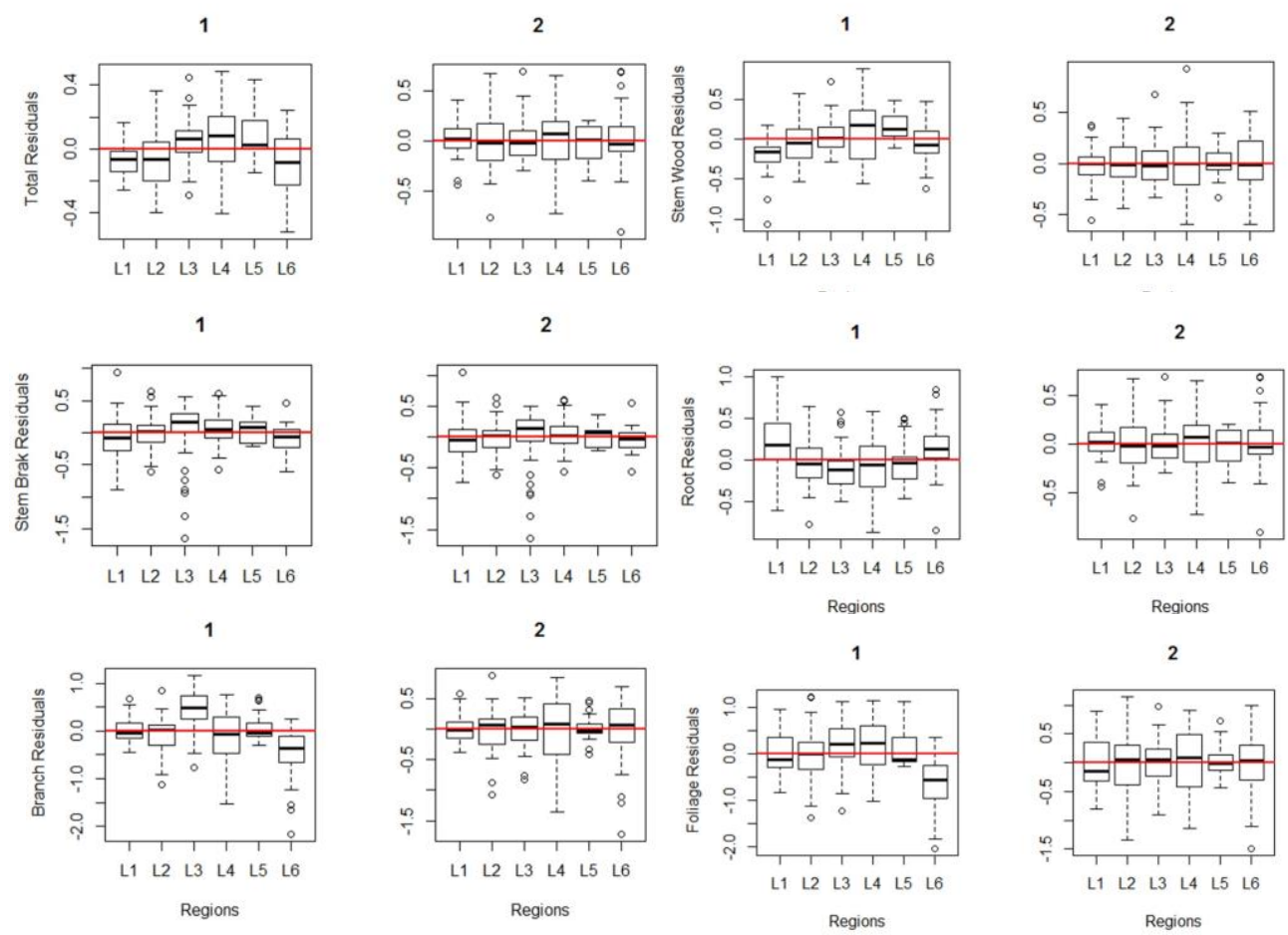

Figure 5.Residual boxplots comparing the non-hierarchical and hierarchical Bayesian approaches. 1 and 2 represent the non-hierarchical and hierarchical Bayesian approaches, respectively.

\section{Discussion}

Theoretical Bayesian methods have been gradually adapted to plant biomass estimations $[9,10,15,16]$. Frequentist statistics assume that parameters are fixed, unknown constant values, whereas Bayesian statistics assume that parameters follow a statistical distribution. For example, Mauricio et al. [9] demonstrated that parameters were well represented by a bivariate normal distribution in an allometric biomass model. One advantage of the Bayesian approach is the MCMC algorithm $[18,28,38]$, which avoids many of the approximations used by the frequentist method [39,40], improving the parameter estimation and model fit.

We establishedtotal tree and biomass component models using non-hierarchical and hierarchical Bayesian approaches. We found that the hierarchical approach performed better, and the hierarchical Bayesian approach significantly improved the accuracy of the biomass model, except for the stem bark model. The stem bark biomass may have accounted for a sufficiently small proportion of the total tree biomass.In general, the hierarchicalapproachperformed better and incorporated the effects of sampling location variability, tree density and other variable factors related to the model-fitting process.

Developing biomass models at large regional scales and improving model accuracy is a significant issue in forest biomass research. Mixed effects models and dummy variable methods are often used to improve the goodness of fit of biomass models [41,42]. When regional effects are present, hierarchical Bayesian approach can be applied to fit the biomass model. Because the data were collected from various spatial regions, biomass model parameters may regionally vary. These variations indicate that regional effects play important roles in the model-fitting process and may be related to unique regional characteristics, such as climate factors,standdensity, tree species or other less noticeable characteristics. The hierarchical approach may yield more realistic results when data are collected at large and spatially variable regional scales. By estimating the total tree and biomass component model variables with this approach, and combined with forest survey data, we can estimate the total and component biomass of stands from each region.

Our results indicate that the hierarchical Bayesian approach improved the model-fitting results, but additional studies may be required to further investigate the effectiveness of the hierarchical 
Bayesian approach for other species and in other regions. Future studies may also be required to confirm that this method is significantly better than the non-hierarchical approach. Note that, if the model fitting process accounts for species differences as a nested factor based on regional differences, the model fitting results should improve, and the hierarchical Bayesian approach would be more effective than the non-hierarchical method.

\section{Conclusions}

The larch biomass data were collected from different regions, including Hubei, Gansu, Hebei, Liaoning, Heilongjiang and Inner Mongolia, which encompass large climate, larch species, silviculture and stand density variations that affect biomass accumulation. These different biotic and abiotic factors introduce variabilities to the larch biomass model, suggesting that allometric equation parameters are better represented by probability distributions rather than fixed values. Therefore, a hierarchical Bayesian approach with informative priors is more suitable for fitting biomass models with regional variations. In this paper, we applied non-hierarchical and hierarchical Bayesian approaches to establish tree biomass models for larch plantations in six Chinese regions. Based on the fitting results, the total tree, root, stem wood, stem bark, branch and foliage biomass model relationships were significant $(p$-values $<0.001)$ for both the non-hierarchical and hierarchical Bayesian approaches.The hierarchical Bayesian approach increased the goodness-of-fit statistics compared to the non-hierarchical approach,significantly improving the accuracy of the biomass model (except for the stem bark) and providing an effective method for estimating larch biomass at the regional scale.

Supplementary Meteraials: The following are available online at http://www.mdpi.com/1999-4907/7/1/18, Table S1: $a$ and $b$ values of 36 biomass equations (total, root, stem wood, stem bark, branch, and foliage biomass) in 6 reported literature for larch in China.

Acknowledgments: This research was supported by the National Natural Science Foundation (No. 31430017 and No.31300536). Numerous researchers assisted with the data collection, but we particularly thank the staff of the Changlinggang Forest Farm, the Xiaolongshan Research Institute of Forestry, the MulanWeichang National Forestry Administration Bureau, the Dagujia Forest Farm, the Mengjiagang Forest Farm and the Wuerqihan Forestry Bureau. We also thank Y.C. Lei for his constructive suggestions and helpful comments regarding the manuscript.

Author Contributions: D.C. and S.Z. conceived and designed the experiments; D.C. and X.H. performed the statistical analysis; and X.S., X.H., D.C. and W.M. contributed materials and analysis tools. D.C. wrote the paper.

Conflicts of Interest:The authors declare no conflict of interest.

\section{References}

1. China Forestry Bureau. The Eighth Forest Resource Survey Report; Chinese Forestry Press:Beijing, China, 2014; p. 18.

2. Ares, A.; Fownes, J.H. Comparisons between generalized and specific tree biomass functions as applies to tropical ash (Fraxinusuhdei). New For. 2000, 20, 277-286.

3. Sah, J.P.; Ross, M.S.; Koptur, S.; Snyder, J.R. Estimating aboveground biomass of broadleaved woody plants in the understory of Florida Keys pine forests. For. Ecol. Manag. 2004, 203, 319-329.

4. Usuga, J.C.; Toro, J.A.R.; Alzate, M.V.R.; Tapias, A.J. Estimation of biomass and carbon stock in plants, soil and forest floor in different tropical forests. For. Ecol. Manag. 2010, 260, 1906-1913.

5. Jenkins, J.C.; Chojnacky, D.C.; Heath, L.S.; Birdsey, R.A. National-scale biomass estimators for United States tree species. For. Sci. 2003, 49, 12-35.

6. Zianis, D.; Muukkonen, P.; Makipaa, R.; Mencuccini, M. Biomass and stem volume equations for tree species in Europe. Silva Fenn. Monogr. 2005, 4, 1-63.

7. Navar, J. Biomass component equations for Latin American species and groups of species. Ann. For. Sci. 2009, 66, 208, doi:10.1051/forest/2009001.

8. Nafus, A.M.; McClaran, M.P.; Archer, S.R.; Throop, H.L. Multispecies allometric models predict grass biomass in semidesert rangeland. Rangel. Ecol. Manag. 2009, 62, 68-72. 
9. Mauricio, Z.C.; Carlos, A.S.; Lauren, A. Probability distribution of allometric coefficients and Bayesian estimation of aboveground tree biomass. For. Ecol. Manag. 2012, 277, 173-179.

10. Zhang, X.Q.; Duan, A.G.; Zhang, J.G. Tree biomass estimation of Chinese fir (Cunninghamialanceolata) based on Bayesian method. PLOS ONE 2013, 8, e79868, doi:10.1371/journal.pone.0079868.

11. Zianis, D.; Mencuccini, M. On simplifying allometric analyses of forest biomass. For. Ecol. Manag. 2004, 187, 311-332.

12. Zianis, D. Predicting mean aboveground forest biomass and its associated variance. For. Ecol. Manag. 2008, 256, 1400-1407.

13. Chesson, P. Mechanisms of maintenance of species diversity. Annu. Rev. Ecol. Syst. 2000, 31, 343-366.

14. Melbourne, B.A.; Hastings, A. Extinction risk depends strongly on factors contributing to stochasticity. Nature 2008, 454, 100-103.

15. Fox, G.A.; Kendall, B.E. Demographic stochasticity and the variance reduction effect. Ecology 2002, 83, 1928-1934.

16. Pfister, C.A.; Stevens, F.R. Individual variation and environmental stochasticity: Implications for matrix model predictions. Ecology 2003, 84, 496-510

17. Gilks, W.R.; Thomas, A.; Spiegelhalter, D.J. A language and program for complex Bayesian modelling. Statistician 1994, 43, 169-178.

18. Cowles, M.K.; Carlin, B.P. Markov chain Monte Carlo convergence diagnostics: A comparative review. J. Am. Stat. Assoc. 1996, 91, 883-904.

19. Myers, R.A.; Mertz, G. Reducing uncertainty in the biological basis of fisheries management by meta-analysis of data from many populations: A synthesis. Fish. Res. 1998, 37, 51-61.

20. Harley, S.J.; Myers, R.A.; Barrowman, N.J.; Bowen, K.G.; Amiro, R.E. Estimation of research trawl survey catchability for biomass reconstruction of the eastern Scotian Shelf. Res. Doc. 2001, 84, 1-53.

21. Shelton, J.H., Ransom, A. Hierarchical Bayesian models of length-specific catchability of research trawl surveys. Can. J. Fish. Aquat. Sci. 2001, 58, 1569-1584.

22. Spiegelhalter, D.J.; Best, N.G.; Carlin, B.P.; Linde, A.V.G. Bayesian measures of model complexity and fit. J. R. Stat. Soc. 2002, 64, 583-640.

23. Green, E.J.; Strawderman, W.E. A comparison of hierarchical Bayes and empirical Bayes methods with a forestry application. For Sci. 1992, 38, 350-366.

24. Finley, A.O.; Kittredge, D.B. Thoreau, Muir and Jane Doe: Different types of private forest owners need different kinds of forest management. North. J. Appl. For. 2006, 23, 27-34.

25. Zhang, X.Q.; Zhang, J.G.; Duan A.G. A hierarchical bayesian model to predict self-thinning line for Chinese fir(Cunninghamialanceolata) in southern China. PLoS ONE 2015, 10, e0139788.

26. Gelman, A.; Hill, J. Data Analysis Using Regression and Multilevel/Hierarchical Models; Cambridge University Press: Cambridge, UK, 2006; p. 58.

27. Gelman, A. Analysis of variance: Why it is more important than ever. Ann. Stat. 2005, 33, 1-53.

28. Baayen, R.H.; Davidson, D.J.; Bates, D.M. Mixed-effects modeling with crossed random effects for subjects and items. J. Mem. Lang. 2008, 59, 390-412.

29. Carlin, B.P.; Louis, T.A. Bayes and Empirical Bayes Methods for Data Analysis;Monographs on Statistics and Applied Probability; Chapman\& Hall: London, U.K, 1996; Volume 69, p.56.

30. Heien, D.M. A note on log-linear regression. J. Am. Stat. Assoc. 1968, 63, 1034-1038.

31. Ter-Mikaelian, M.T.; Korzukhin, M.D. Biomass equations for sixty-five North American tree species. For. Ecol. Manag. 1997, 97, 1-24.

32. Overman, J.P.M.; Witte, H.J.L.; Saldarriaga, J.G. Evaluation of regression models for above-ground biomass determination in Amazon rainforest. J. Trop. Ecol. 1994, 10, 207-218.

33. Wagner, H.; Tüchler, R. Bayesian estimation of random effects models for multivariate responses of mixed data. Comput. Stat. Data Anal. 2010, 54, 1206-1218.

34. Bates, D.M.; Watts, D.G. Nonlinear Regression Analysis and Its Applications; John Wiley \& Sons: New York, NY, USA,1988; p. 102.

35. Barrio-Anta, M.; Balboa-Murias, M.; Castedo-Dorado, F.; Diéguez-Aranda, U.; Alvarez-González, J.G. An ecoregional model for estimating volume, biomass and carbon pools in maritime pine (Pinuspinaster Ait) stands in Galicia (northwestern Spain). For. Ecol. Manag. 2006, 223, 24-34.

36. Hadfield, J.D. MCMC methods for multi-response generalized linear mixed models: The MCMCglmm R package. J. Stat. Softw. 2010, 33, 1-22. 
37. Sturtz, S.; Ligges, U.;Gelman, A. R2WinBUGS: A package for running WinBUGS from R. J. Stat. Softw. 2005, 12, 1-16.

38. Carlin, B.P.; Clark, J.S.; Gelfand, A.E. Hierarchical Modelling for the Environmental Sciences: Statistical Methods and Applications; Oxford University Press: Oxford, UK, 2006; p. 103.

39. Paap, R. What are the advantages of MCMC based inference in latent variable models. Stat. Neerl. 2002, 56, 2-22.

40. MarcKéry. Introduction to WinBUGS for Ecologists: A Bayesian Approach to Regression, ANOVA, Mixed Models and Related Analyses; Library of Congress Cataloging: Washington, D.C., USA, 2010; p. 8.

41. Cole, T.G.; Ewel, J.J. Allometric equations for four valuable tropical tree species. For. Ecol. Manag. 2006, 229, 351-360.

42. Pérez-Cruzado, C.; Rodríguez-Soalleiro, R. Improvement in accuracy of aboveground biomass estimation in Eucalyptus nitens plantations: Effect of bole sampling intensity and explanatory variables. For. Ecol. Manag. 2011, 261, 2016-2028.

(C) 2015 by the authors; licensee MDPI, Basel, Switzerland. This article is an open access article distributed under the terms and conditions of the Creative Commons by Attribution (CC-BY) license (http://creativecommons.org/licenses/by/4.0/). 